Bulletin d'études orientales

\title{
Grammaticalisation du mașdar kawn en arabe contemporain : « le fait de », " parce que », « que »
}

\section{Catherine Pinon}

\section{OpenEdition}

\section{Journals}

Édition électronique

URL : http://journals.openedition.org/beo/4829

DOI : $10.4000 /$ beo.4829

ISSN : 2077-4079

Éditeur

Presses de l'Institut français du Proche-Orient

Édition imprimée

Date de publication : 1 avril 2017

Pagination : $77-100$

ISBN : 978-2-35159-731-6

ISSN : 0253-1623

Référence électronique

Catherine Pinon, «Grammaticalisation du masdar kawn en arabe contemporain : « le fait de », "parce que », «que » », Bulletin d'études orientales [En ligne], LXV | 2017, mis en ligne le 01 avril 2019, consulté le 06 janvier 2021. URL : http://journals.openedition.org/beo/4829 ; DOI : https://doi.org/10.4000/beo. 4829 


\section{Grammaticalisation du mașdar kawn en arabe contemporain : « le fait de », « parce que », « que »}

Catherine PINON

Résumé : Cet article s'intéresse à l'emploi dit «grammatical » du mașdar kawn. Après avoir fait un état des lieux des mentions de cet emploi dans les grammaires arabes et dans les grammaires orientalistes ou arabisantes, on y propose une description exhaustive fondée sur corpus des différents emplois de kawn en arabe contemporain. Les différentes valeurs que sont « le fait d'être », " parce que » voire l'emploi comme simple subordonnant « que », suggèrent la grammaticalisation de kawn.

Mots-clés : arabe contemporain, syntaxe, grammaire arabe, grammaticalisation, mașdar, kawn, subordonnant, complément de cause, «parce que ».

Abstract : This paper deals with the uses of kawn in contemporary Arabic. After an overall view of the indications of how this form is used in the Arabic grammar and the Orientalist or Arabist grammars, this paper gives a comprehensive description corpus based on the different uses of kawn in contemporary Arabic. The different values like "being", "because" or the subordinator "that" suggest the grammaticalization of kawn.

Keywords : contemporary arabic, syntax, arabic grammar, grammaticalization, mașdar, kawn, subordinator, "because".

$$
\begin{aligned}
& \text { ملخص : تُعنى هذه الهقالة بدراسة استخدامات مصدر الفعل الناقص \اكان | في اللغة العربية كأداة }
\end{aligned}
$$

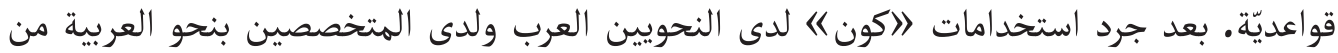

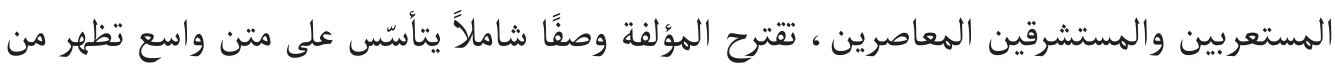

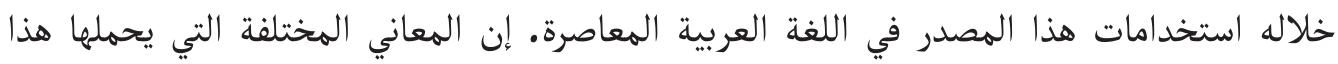

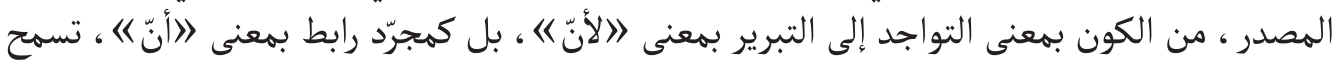

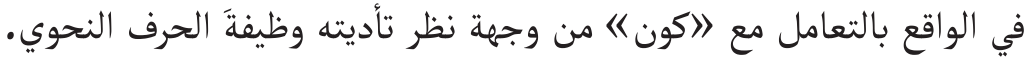

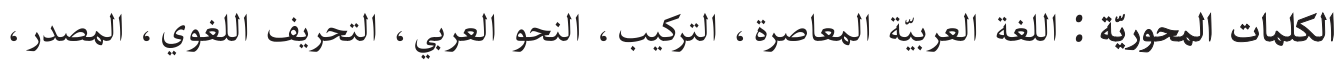

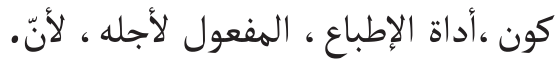


Qui fréquente les grammaires dites d'arabe moderne n'aura pas manqué de constater que celles-ci sont bien souvent davantage une transcription du contenu des grammaires de l'arabe classique que de réelles grammaires décrivant l'état moderne de l'arabe. Partant de ce constat, il nous a semblé nécessaire d'apporter une contribution à la grammaire de l'arabe moderne en proposant des fragments de syntaxe de l'arabe contemporain. À moyen terme, l'objectif est d'établir une grammaire adéquatement descriptive de cet état. C'est dans cette entreprise de description quantifiée et systématique de la syntaxe de l'arabe contemporain, fondée sur corpus, que s'intègre cet article. ${ }^{1}$

Le nom verbal (mașdar) kawn est connu pour son sens lexical signifiant « univers ", mais admet aussi un emploi que l'on peut qualifier de grammatical signifiant «le fait d'être ». Ce second emploi ne figure pour ainsi dire pas dans les grammaires arabes traditionnelles, bien qu'il soit régulièrement employé par les grammairiens eux-mêmes. Une étude à même de décrire cet emploi relativement courant dans la langue moderne fait actuellement défaut. Elle seule, pourtant, est susceptible de nous aider à comprendre le processus de grammaticalisation qui permet de passer du «fait de » à « parce que », voire à un simple marqueur de subordination comme « que ».

Dans un premier temps, nous synthétiserons les mentions faites de cet emploi dans les grammaires arabes et orientalistes ou arabisantes. Ensuite, à partir d'un corpus d'arabe contemporain, nous observerons la syntaxe propre à l'utilisation de kawn, ainsi que les différentes valeurs portées par ce mașdar, afin de proposer une hypothèse quant à sa grammaticalisation.

\section{Kawn dans les grammaires}

\section{Sources arabes}

Kawn constitue pour les grammairiens un outil qu'ils utilisent régulièrement pour exprimer l'état de fait ou la cause. Nous pouvons citer ce passage où figure une occurrence d'ailleurs en coordination avec li-'anna (parce que) parmi les innombrables occurrences que contient al-Imlà' 'alā l-Käfiya fìl-naḥw d'Ibn al-Hāğib ${ }^{2}$ :

(1) fa-'in șaḥha hurūüg "yadribu" 'an al-hadd li-kawni-hi dalla 'alā 'ahad al-'azmina fa-l-yașiḥh hurūüğ "ḍarib" 'an al-hadd li-'anna-hu dalla 'alā' ahad al-'azmina wa-'in kānat dalālatu-hu muštaraka.

S'il est exact d'exclure yadribu (il frappe) de la catégorie [du nom] du fait qu'il réfère à l'un des trois temps, alors il convient d'exclure dạrib (frappant, qui frappe) de ladite catégorie parce qu'il réfère à l'un des trois temps, même si sa signification est partagée [entre le présent et le futur].

1. Sur les fondements théoriques de cette entreprise, notamment la notion d'accrétion dans les grammaires arabes et le concept de sui-langue arabe classique, voir Pinon (2011,2012a). Sur d'autres éléments de description déjà effectués concernant le verbe kāna, voir PINON 2012 b, 2013 b.

2. Ibn al-Ḥāğib, Al-Imlä’ 'alā l-Kāfiya fì l-naḥw, folio $2 b$. 
Certains auteurs utilisent kawn très régulièrement dans leurs explications, comme Abū al-Barakāt al-Anbārī ${ }^{3}$, notamment dans les titres en complément du nom 'illa (raison, cause) où il est ensuite paraphrasé par 'an yakūn (qu'il soit) :

(2) 'illat kawn al-tamyīz nakira. [...] Fa-lima wağiba 'an yakūn al-tamyīz nakira?

La raison du fait que le complément spécificatif est indéterminé. [...] Alors pourquoi le spécificatif doit-il être indéterminé ? (lit. pourquoi faut-il que soit le spécificatif indéterminé).

Cet emploi n'est donc ni rare, ni récent; il est attesté en nombre dans des textes bien antérieurs à la période moderne. Cependant, les grammairiens qui mentionnent l'emploi particulier de ce mașdar sont rares ${ }^{4}$ et se bornent à expliquer qu'en tant que mașdar du verbe kāna bivalent (kawn mașdar kāna l-nāqișa), il a besoin d'un ism et d'un habar. Ibn 'Aqūl, dans son commentaire de la Alfiyya d'Ibn Mālik, semble être le seul auteur à commenter systématiquement les emplois de kawn. Al-Ġalāyīnī (1973, p. 279-280) cite quant à lui trois exemples, les deux premiers forgés, le troisème normalement puisé dans la tradition poétique arabe, mais il ne les discute que du point de vue de l'írāb:

(3) kawnu-ka muğtahidan hayrun la-ka.

Que tu sois appliqué est mieux pour toi, lit. le fait que tu sois.

(4) kawnu l-rağul taqiyyan hayrun la-hu.

Que l'homme soit pieux est mieux pour lui, lit. le fait que.

(5) bi-baḍlin wa-hilmin sāda fĩ qawmi-hi l-fatā/wa-kawnu-ka 'iyyā-hu 'alay-ka yasīru

Par l'abnégation et la longanimité, le jeune preux est le seigneur de son clan/et du fait que tu es doué, il t'est facile d'en être un.

On trouve aussi mention de cet emploi de kawn dans le Munğid de la langue contemporaine ${ }^{5}$ :

(6) kawn : maș./kāna : «'uhliya sabïlu-hu li-kawni-hi mağnūnan. »

Le fait d'être : nom d'action/être : « il a été relâché parce qu'il est fou ».

Il convient donc de noter que l'emploi de kawn seul ou en composition avec d'autres particules dans un tour exprimant la cause ou la raison n'est pas récent mais qu'il n'apparaît que sporadiquement dans les grammaires arabes traditionnelles.

3. Al-Anbārī, Kitāb asrār al-'arabiyya, I, p. 155 pour l'exemple.

4. Brève mention en est faite chez Al-Anbārī, Kitāb asrār al-'arabiyya; Ibn 'Aqūl, Šarh Ibn 'Aqūl 'alā Alfiyya Ibn Mālik; Ibn Hišām al-Anșārī, Mūğaz; al-Ġalāyīnī, Ǧāmi' al-durūs al-'arabiyya; Ḥ. 'ABBĀs, Al-Naḥw al-wāfì. Dans les grammaires suivantes, nous n'avons pas trouvé mention du maṣdar kawn en tant que tel : al-Anșārī, Muġnī l-labīb

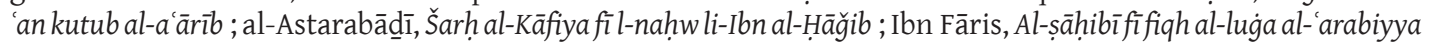
wa-sunan al-'arab fì kalāmihāa; Ibn Fāris, Muğmal al-luġa; Ibn Mālik, Šarh al-kāfiya al-šāfiya; al-Mubarrad, Al-Muqtadab ; al-Mubarrad, Al-Kāmil fīl-luġa wa-l-adab; Sībawayhi, Al-Kitāb̀ ; al-Zamahššrī, Al-mufașșal fì 'ilm al-'arabiyya. Tous ces ouvrages sont accessibles en ligne sur le site d'al-Maktaba al-šāmila (http://shamela.ws).

5. Al-Munǧid fì l-luġa l-mu'āsirira, p. 1258 a. 


\section{Sources orientalistes et arabisantes}

Silvestre de Sacy (1810, p. 299-300) indique, dans la droite ligne des grammairiens arabes, que « le nom d'action du verbe kāna est très souvent employé, comme le verbe lui-même, avec un sujet et un attribut, le sujet étant mis au génitif, sous forme de complément d'un rapport d'annexion, et l'attribut à l'accusatif ». Il cite les exemples suivants :

(7) li-kawni-ka qawiyyan wa-kawni gayri-ka da îfan.

Parce que tu es fort et que les autres sont faibles.

(8) min kawni-hi qad wağada-hà 'alā l-țarīq.

Parce qu'il l'avait trouvée sur le chemin.

(9) min kawni-him lā ya'rifūna 'ayyunā fa' ala hād̄ā.

Parce qu'ils ne savoient [savaient] pas qui de nous avoit [avait] fait cela.

Dans ces trois exemples, la combinaison de kawn avec les prépositions li- ou min sert clairement à exprimer la cause. Silvestre de Sacy traduit d'ailleurs les trois occurrences par «parce que ». Mais pour lui, kawn garde dans (8) et (9) un rôle d'exposant temporel puisqu'il déclare: «nous avons observé que le verbe kāna, joint à l'aoriste ou au prétérit d'un autre verbe, donne au premier de ces temps la valeur de l'imparfait, et au second la valeur du plusqueparfait. Ajoutons ici que le nom d'action du verbe kāna peut être employé de la même manière » (SILVESTRE DE SACY 1810, II, p. 299-300). Pour nous, l'emploi de kawn est totalement « détemporalisé ». Dans (8), c'est l'emploi de qad qui exprime l'antériorité de l'action; dans (9), on pourrait tout à fait traduire par «parce qu'ils ne savent pas qui de nous a fait cela ». Si nous avions accès au contexte précédent l'occurrence et qu'on y trouvait un verbe à l'accompli, ce serait cet accompli qui permettrait d'interpréter lā yárifūna comme un plus-que-parfait. La proposition livrée dans un contexte plus large nous permettrait donc de comprendre pourquoi Silvestre de Sacy a traduit «lā yárifüna " par un imparfait, mais en l'absence d'un tel contexte, il nous semble pouvoir affirmer que kawn n'apporte aucune valeur temporelle dans ces deux exemples. ${ }^{6}$

Pour W. Fischer, "kawnun "être" est utilisé en arabe post-classique pour intégrer des propositions dans les phrases complexes. Le sujet de la proposition subordonnée apparaît comme un génitif de kawnun, et le prédicat qui suit est à l'accusatif ou comme une proposition complément de manière hăl » (FISCHER 2002, § 416, Rq. 3). Il donne les deux exemples suivants ${ }^{7}$ :

6. Nous avons constaté que dans les grammaires, le contexte des exemples cités est réduit au minimum, ce qui pose souvent des problèmes d'interprétation. D'autre part, le fait que les exemples ne soient pas référencés ne permet pas à l'usager des grammaires d'accéder à ce contexte. Dans notre projet de grammaire descriptive de l'arabe contemporain, les outils actuels nous aident à combler ces problèmes de contexte et de référencement.

7. Lorsque nous citons des exemples provenant de sources anglaises, nous reproduisons d'abord la traduction anglaise, avant de traduire en français. 
(10) mana'a-hu 'ani l-rihllati kawnu-hu ğabānan.

The fact that he is a coward prevented him from making the journey.

Le fait qu'il est lâche l'a empêché de voyager.

(11) ğăàa l-nāsu 'ilay-hi li-l-hanā̉i li-kawni l-wazīri kallama-hu.

The people came to congratulate him, because the vizier had spoken to him.

Les gens sont venus le voir pour le féliciter parce que le vizir lui avait parlé.

Si l'on se borne là encore à expliquer simplement le fonctionnement du syntagme [kawn + complément], il faut relever que Fischer traduit (10) par « the fact that » (le fait que) et (11) par « because » (parce que). Il atteste donc de l'existence de deux emplois distincts de kawn: employé directement, il signifierait plutôt «le fait de », mais en combinaison avec une particule comme li-, il signifierait clairement la cause « parce que ».

On retrouve cet emploi en arabe moderne chez E. Badawi, M. Carter et A. Gully (2004, p. 402-403) qui en expliquent le fonctionnement, en notant que le nom verbal a la même syntaxe que la forme verbale, pouvant introduire tant un syntagme nominal qu'un syntagme verbal :

(12) kawnu-ka șadīqan.

Your being a friend.

En tant qu'ami.

(13) kawnu-nā naḥtāğu 'ilā l-istițmāri l-hāriğìi.

Our being in need of external investment, lit. our being we need.

Du fait de notre besoin d'investissements extérieurs, lit. le fait que nous avons besoin.

(14) fỉ kawni hādāa l-inğāzi yumațitilu -mtidādan hallāqan.

In that this achievement represents a creative extension, lit. in the being of this achievement it represents.

En ce que cette réalisation représente une extension créative, lit. en le fait que.

(15) li-kawni-him 'adrakū tabíat al-ma'raka.

Because they had grasped the nature of the conflict, lit. their being they grasped.

Parce qu'ils ont saisi la nature du conflit.

Ces exemples nous permettent de mieux appréhender les différents emplois de kawn: du simple «fait d'être » $(12,13)$, on passe au «fait que » (14) pour arriver à marquer clairement la cause, « parce que » (15), en composition avec la particule li-.

En résumé, si l'emploi grammatical de kawn apparaît dans quelques grammaires, les exemples fournis n'appellent aucun commentaire quant à l'évolution de l'emploi de kawn. Or, celle-ci nous semble intéressante à étudier. 


\section{Corpus : présentation des occurrences}

Pour faire le point sur les emplois grammaticaux de ce mașdar en arabe moderne, nous avons décidé de l'observer systématiquement dans un corpus d'arabe contemporain, afin d'en fournir une description et d'en classifier les différents emplois. Le corpus compte 1470000 mots et regroupe des textes produits entre 2002 et 2011, provenant de sept pays arabes (Maroc, Tunisie, Égypte, Syrie, Liban, Arabie Saoudite, Yémen) et ressortissant à trois genres (littérature, presse, blogs). En nous référant au principe de saturation délimité pour l'arabe par M. Van Mol (2003) aux alentours de 200000 mots, ce corpus est suffisant pour atteindre une description satisfaisante de la langue puisqu'il regroupe plus de 210000 mots pour chaque pays et près de 500000 mots par genre ${ }^{8}$. Même s'il s'agit d'un corpus en synchronie, il permet d'accéder aux différentes valeurs de kawn et amène à proposer des hypothèses quant à sa grammaticalisation. Une comparaison en diachronie permettrait par la suite de valider ces hypothèses.

Le corpus contient 463 occurrences du mașdar kawn. Celui-ci peut être employé soit dans un sens lexical avec le sens d' " univers ", soit comme outil grammatical. Nous mentionnons dans le tableau ci-dessous le total des occurrences, suivi entre parenthèses des décomptes respectifs pour l'emploi lexical (186 occurrences en tout) puis pour l'emploi grammatical (277 occurrences au total).

\begin{tabular}{|l|l|l|l|l|}
\cline { 2 - 5 } \multicolumn{1}{c|}{} & Blogs & Littérature & Presse & TOTAL \\
\hline Arabie Saoudite & $45(26 / 19)$ & $17(14 / 3)$ & $16(0 / 16)$ & $78(40 / 38)$ \\
Égypte & $24(8 / 16)$ & $13(7 / 6)$ & $4(0 / 4)$ & $41(15 / 26)$ \\
\hline Liban & $21(8 / 13)$ & $20(9 / 11)$ & $16(1 / 15)$ & $57(18 / 39)$ \\
\hline Maroc & $27(4 / 23)$ & $9(7 / 2)$ & $28(0 / 28)$ & $64(11 / 53)$ \\
\hline Syrie & $38(6 / 32)$ & $45(37 / 8)$ & $26(2 / 24)$ & $109(45 / 64)$ \\
\hline Tunisie & $8(4 / 4)$ & $23(20 / 3)$ & $3(2 / 1)$ & $34(26 / 8)$ \\
\hline Yémen & $43(26 / 17)$ & $9(1 / 8)$ & $28(4 / 24)$ & $80(31 / 49)$ \\
TOTAL & $206(82 / 124)$ & $136(95 / 41)$ & $121(9 / 112)$ & $463(186 / 277)$ \\
\hline
\end{tabular}

Tableau 1: Répartition des occurrences du mașdar par genre et par pays.

Il apparaît très clairement que l'emploi grammatical est plus important que l'emploi lexical. En effet, $60 \%$ des occurrences du mașdar sont d'un emploi grammatical, contre $40 \%$ d'un emploi lexical. L'emploi grammatical de kawn représente 0,019\% des mots de notre corpus. Ces chiffres peuvent être partiellement comparés à ceux fournis par T. Buckwalter et D. Parkinson (2011) dans leur dictionnaire classé par fréquences, où kawn apparaît au 739e rang (ex cequo) avec le mot mas'ala (question) figurant à la 738e place. Kawn est présent dans $97 \%$ des textes de leur corpus de 30 millions de mots. Ils y ont relevé 5136 occurrences

8. À ce sujet, voir PINON 2012 b et 2013 a. 
du mot kawn, soit $0,017 \%$ du total de leur corpus ${ }^{9}$. Évidemment, ils ne mentionnent pas la répartition entre emploi lexical et emploi grammatical, mais l'exemple qu'ils fournissent révèle certainement, comme c'est le cas dans notre corpus, une prédominance de ce dernier. En effet, voilà comment est présentée cette entrée du dictionnaire :

« kawn vn. Being, by virtue of being; li-kawn because; n. al-kawn the universe, existence. la yamna' kawn al-'as'ila șa'ba min 'an tabhat 'ani l-'iğāba. - The fact that the questions are hard does not prevent one from searching for the answers. » kawn nom verbal : être, le fait d'être ; li-kawn parce que ; al-kawn substantif : l'univers, existence.

(16) lā yamna 'kawn al-'as'ila șa'ba min 'an tabhat 'ani l-'iğāba.

Le fait que les questions soient difficiles ne doit pas t'empêcher de chercher les réponses.

Il est très intéressant de constater que les auteurs ont clairement mentionné la différence entre l'emploi comme nom verbal (ce qui correspond à notre emploi grammatical, au mașdar du verbe kāna bivalent) et comme substantif (mașdar du verbe kāna monovalent ${ }^{10}$. De même, ils citent li-kawn comme synonyme de " parce que ${ }^{11}$ ".

Si nous étudions maintenant la répartition des deux types d'emploi par genre, on constate que l'emploi lexical est rare dans la presse et que c'est dans la littérature que l'emploi grammatical est le moins représenté, comme cela apparait clairement dans le tableau 2 ci-dessous :

\begin{tabular}{|l|c|c|}
\cline { 2 - 3 } \multicolumn{1}{c|}{} & Emploi lexical & Emploi grammatical \\
\hline Blogs & $40 \%$ & $60 \%$ \\
\hline Littérature & $70 \%$ & $30 \%$ \\
\hline Presse & $7 \%$ & $93 \%$ \\
\hline
\end{tabular}

Tableau 2: Répartition des emplois lexicaux et grammaticaux du mașdar par genre.

Une remarque s'impose pour préciser la méthode qui a été la nôtre. Pour extraire automatiquement les occurrences du mașdar kawn de notre corpus, nous avons utilisé le

9. Nous avons relevé près du double d'occurrences si nous totalisons les emplois grammaticaux et lexicaux ( $0,031 \%$ des mots de notre corpus). Le corpus de Buckwalter et Parkinson étant vingt fois plus gros que le nôtre, on peut supposer, en se référant à la loi des grands nombres, que les emplois de kawn sont quelque peu surreprésentés dans notre corpus. Pour avoir un ordre d'idée de la fréquence des mots en arabe, en nous basant toujours sur le dictionnaire de Buckwalter et Parkinson, rappelons que l'article est employé à hauteur de 16,7 \% ( $\left.1^{\mathrm{er}} \mathrm{mot}\right)$, la conjonction wa à $3,7 \%$ ( $\left.2^{\mathrm{e}} \mathrm{mot}\right)$, qu'au $10^{\mathrm{e}}$ mot nous passons sous le seuil des $1 \%$. Le $5000^{\mathrm{e}}$ mot par ordre de fréquence est le substantif d̦amm (adjonction, rattachement) avec 0,00056\%. Les 40 premiers mots sont, à quelques exceptions près, des particules et des pronoms.

10. Nous opposons au verbe kāna bivalent (kāna al-nāqișa dans la tradition grammaticale arabe) le verbe kāna monovalent (kāna al-tāmma dans la tradition grammaticale arabe).

11. Nous verrons plus bas que li-kawn est en effet bien plus employé que bi-kawn (de l'ordre d'un rapport de plus de deux tiers à moins d'un tiers). 
logiciel de textométrie Lexico ${ }^{12}$. Nous avons recherché la séquence kawn en arabe dans notre corpus et supprimé de la liste des résultats tout ce qui apparaissait comme n'étant pas le mașdar (principalement des verbes ayant pour troisième radicale [k] conjugués au masculin pluriel comme yudrikūn ou yumsikūn). Ensuite, nous avons passé en revue la concordance et regardé le reste des occurrences une à une pour déterminer si l'emploi était lexical ou grammatical. Ce qui nous intéressait, c'était d'obtenir la liste de toutes les occurrences grammaticales de kawn, de manière à étudier en détail ce type d'emploi. La question de savoir comment, formellement, il est possible de déterminer si l'emploi de kawn est lexical ou grammatical n'a pas été posée. ${ }^{13}$

Sur les 277 occurrences comptabilisées, certaines ont été écartées pour l'étude de détail, provenant d'articles de presse reversés ou de billets de blogs en double, ainsi que quelques occurrences tirées d'un registre totalement dialectal. C'est pourquoi les pourcentages qui suivent sont relatifs à 268 occurrences d'un emploi grammatical du mașdar kawn.

\section{Analyse des constructions avec kawn d'emploi grammatical}

En tant que mașdar du verbe kāna, kawn a les propriétés du nom en arabe et conserve la structure argumentale du verbe kāna dont il est issu ${ }^{14}$. Dans son emploi grammatical, kawn est toujours issu du verbe kāna bivalent : il aura donc deux compléments, le premier étant la personne ou la chose ayant une certaine qualité et le second étant la qualité en question. Kawn est en état construit avec le premier complément (que nous pouvons nommer par analogie ism kawn) ${ }^{15}$ et suivi du second complément (que nous pouvons nommer habar kawn $)^{16}$. De ce fait, le ism kawn sera au cas indirect puisque complément du nom kawn et le habar kawn au cas direct. ${ }^{17}$

12. http://www.tal.univ-paris3.fr/lexico/.

13. À ce sujet, D. E. Kouloughli nous faisait remarquer qu'il était dommage de ne pas davantage expliciter la démarche suivie pour sélectionner les exemples pertinents, empêchant ainsi de rapporter les chiffres avancés à des données plus globales. Il prenait l'exemple de la séquence kawnu-nā, ambiguë, puisque pouvant signifier «notre univers» ou « le fait que nous soyons». En cherchant sur Internet, par exemple dans un article de Wikipédia consacré à la cosmologie, on peut observer que toutes les occurrences de kawnu-nā ont un sens lexical. Pour D. E. Kouloughli, une démarche à base de stricte analyse de corpus ne peut pas esquiver la discussion de tels faits, qui ont d'ailleurs l'intérêt de soulever une question simple : comment sait-on, c'est-à-dire à partir de quels indices formels dans un texte, que kawnu-nā est ou n'est pas à interpréter comme un substantif?

14. S'il conserve sa structure argumentale, il semble qu'une restriction s'opère naturellement quant à l'ordre des syntagmes dans la phrase. En effet, si le habar kāna peut être antéposé, l'ordre après kawn est nécessairement respecté (premier puis second complément, éventuellement séparés par une incise ou un complément non essentiel), car le premier complément est complément du nom et ne peut pas être séparé de kawn.

15. Celui-ci est d'ailleurs nommé ism al-mașdar al-nāqiṣ par al-Ġalāyīnī (1973, p. 280). Voir ci-dessus.

16. Il semble qu'on puisse trouver là un premier élément permettant de départir automatiquement les emplois lexicaux des emplois grammaticaux de kawn. En effet, seuls les premiers peuvent être déterminés par l'article. Les seconds seront toujours en état construit.

17. Parmi les énoncés où le habar kawn doit porter une marque de cas visible, nous n'avons relevé que quatre emplois fautifs, tous provenant de blogs. 
Quelle que soit la nature de la phrase à subordonner, kawn s'applique à une phrase à tête nominale. Ce qui, dans la phrase enchâssée, était initialement le thème ou le sujet devient le ism kawn; et ce qui tenait lieu de propos ou de complément devient le habar kawn. Logiquement, le ism kawn devrait donc forcément être nominal ou propositionnel, alors que le habar kawn pourrait tout aussi bien être un syntagme nominal, un syntagme prépositionnel ou une phrase verbale.

Dans cette partie, nous allons tout d'abord procéder à une description exhaustive des emplois de kawn en relevant toutes les possibilités d'emploi qui figurent dans le corpus et en en détaillant les caractéristiques syntaxiques. Nous proposerons ensuite une classification des différentes valeurs de kawn pour réfléchir à sa grammaticalisation.

Construction du syntagme

Nature du ism kawn

Kawn est toujours en état construit et peut avoir comme ism un pronom, un nom ou une proposition introduite par 'an ou 'anna (que).

- Kawn suivi d'un pronom :

Kawn est majoritairement en état construit avec un pronom:c'est le cas dans 179 phrases de notre corpus, soit $67 \%$ des emplois grammaticaux de kawn. Si l'on regarde en détail les pronoms utilisés, on s'aperçoit que ce sont ceux de la troisième personne du singulier qui sont les plus employés. Kawn servirait donc plutôt à attribuer une qualité à une chose ou une personne dont on parle, plutôt qu'à soi-même ou à un interlocuteur direct. En effet, seulement $16 \%$ des pronoms sont de la 1re personne (du singulier uniquement: kawn-ī est présent 28 fois dans notre corpus), comme dans l'exemple suivant:

(17) Blog - Syrie - Marcellita

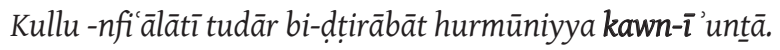

Toutes mes émotions sont dirigées par des perturbations hormonales du fait que je sois une femme.

La deuxième personne est encore moins présente : on ne la rencontre que dans $2 \%$ des cas (kawn-ka et kawn-ki apparaissent deux fois, kawn-kum une seule fois) :

(18) Blog - Maroc - Ijork

bi-l-'idāfa 'ilā kawni-ki kātibat 'amūd, fa-'anti 'ayḍan kātibat sīnāryū wa-muhriğa wa-laday-ki țumūh li-kitābat al-riwāya.

En plus du fait que tu es journaliste, tu écris des scénarios, mets en scène [des films] et as l'ambition d'écrire des romans.

Les pronoms de la 3e personne sont les plus employés ( $82 \%$ des pronoms, avec 66 kawn-hā dans notre corpus, 62 kawn-hu, 12 kawn-hum, 2 kawn-humā et 1 kawn-hunna,

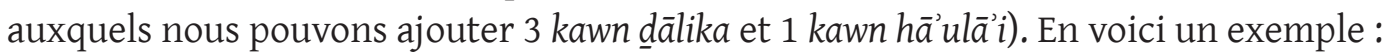


(19) Presse - Liban - Annahar wa-hāàā l-tsunāmī yașil bi-rtifā' ḥawālay mitr wa-niṣf al-mitr 'ilā Bayrūt, kawni-hi yanțaliq min ğanūb Qubruṣ li-'anna-hu kulla-mā ba'adat al-masāfa yaftarid 'an takūn quwwat al-tsunāmī aqall.

Ce tsunami arrivera à une hauteur d'un mètre et demi sur Beyrouth, parce qu'il est parti du sud de Chypre et que plus la distance s'allonge, plus la puissance du tsunami est censée diminuer.

\section{- Kawn suivi d'un nom :}

Si kawn n'est pas en état construit avec un pronom, il l'est avec un nom. C'est le cas dans 84 phrases de notre corpus, soit 31,5\% des emplois grammaticaux de kawn. Il peut s'agir de noms propres ou de substantifs, ainsi que de tout autre terme entrant dans la catégorie du nom en arabe, participes actif et passif notamment.

(20) Presse - Maroc - Attajdid

tamma 'ihbār a'ḍ̄' al-lağna bi-kawn al-wazīra sawfa tatafadḍal bi-'arḍ al-taqrīr fi-ğtimā' al-lağna.

On a informé les membres du comité du fait que la ministre présenterait le rapport lors de la réunion du comité.

\section{- Kawn suivi d'une proposition :}

Plus rarement, kawn peut être construit avec une proposition introduite par les particules an ou anna. Nous n'en avons que quatre exemples dans notre corpus et remarquons qu'à chaque fois, le habar kawn est une phrase verbale.

(21) Blog - Maroc - Ibnabad

'ammā l-ihtilāf bayna-hā fa-yatağassad fì kawn 'annal-ḥurūb al-'ūlā kānat fì 'ahd al-ğāhiliyya, 'ammā l-'ahìra fa-tahdut fí 'ahd al-'islām.

La différence entre elles [ces guerres] se matérialise dans le fait que les premières guerres eurent lieu au temps de la Ğāhiliyya; quant aux dernières, elles survinrent à la période islamique.

(22) Presse - Maroc - Alalam

hal al-taharriyyāt lā tazāl mustamirra li-tahdīid al-mas'ūiliyyāt wa-tartīb al-natā'iğ, li-kawn 'awwalan 'anna l-ihtilās al-mutahaddaț 'an-hu-ktušifa bi-l-șudfa, wa-stamarral-baht fi-hi li-sanawāt qabla 'ihâalat al-qadiyya 'alā l-qad̄à'?

Est-ce que les investigations sont toujours en cours pour déterminer les responsabilités et trier les résultats, du fait, premièrement, que le détournement de fonds dont il est question a été découvert par hasard et les investigations menées au sujet dudit détournement se sont poursuivies pendant des années avant de renvoyer l'affaire devant la justice?

Remarquez que, dans l'exemple précédent, le locuteur a séparé le nom kawn et son complément par l'adverbial 'awwalan « premièrement ». Ce qui permet ceci, à notre avis, c'est que la construction syntaxique avec kawn est toujours la même. 'Awwalan étant tout de suite interprété comme ne pouvant pas être le ism kawn, le lecteur s'attend à trouver celui-ci juste après. Ce fait est fort intéressant et peut être considéré comme une marque de grammaticalisation de kawn: dans cet emploi très grammatical, le fait que kawn introduise 
une proposition elle-même introduite par 'anna (exemples 21 et 22) ou par 'an (23) amène à isoler de plus en plus kawn et à le détacher de son ism. On accepte alors l'introduction d'un terme entre kawn et son ism.

(23) Blog - Egypte - Talmehaty

'inna 'aynayya tazraf [sic] al-dam' daman 'alā kā̉in yantamī li-l-insāniyya bi-l-šakl wa-lā yahmil min-hā siwā l-šakl wa-l-maẓhar fa-qad a'țā-hu -Llāh mawhibat al-qadah wa-l-d̂amm wa-hntilāq al-fitan (wa-matal tilka l-ğarā’im ašadd min al-qatl) wa-laysa a'ḍam [sic] min 'an aqūl « inna li-l-Lāh wa-'inna ilay-hi rāği ūn » li-'anna-hu kawn 'an yakūn al-'insān yatahallā 'an kaynūnati-hi wa-yantamī 'ilā fi'a 'uhrā min al-mahlūqāt d̂ dawāt al-'arba'... mușība.

Je pleure des larmes de sang sur un être qui appartient à l'humanité en forme mais ne porte d'elle que la forme et l'apparence ; Dieu lui a donné le don de dénigrement, de diffamation et de semer la zizanie (crimes qui sont encore pires que le meurtre ${ }^{18}$ ) et il n'y a rien de plus fort que de dire «nous sommes à Dieu et c'est vers lui que nous retournons » parce que le fait qu'un être humain renonce à son humanité et se rattache à une autre catégorie de créatures, celle des quadrupèdes est... une calamité.

Dans cet exemple, on a préféré à une proposition introduite par anna («kawn 'anna l-insān yatahallā 'an kaynūnati-hi») une proposition introduite par an ("kawn 'an yakūna l-'insān... »). L'ensemble produit un effet d'insistance sur le mauvais choix fait par le type d'individu que critique l'énonciateur, selon son point de vue.

Une plongée dans Internet à la recherche de l'expression «kawn an » nous fournit quelques autres attestations, mais relevant semble-t-il majoritairement d'un langage spontané, provenant de forums, de tweets, de commentaires postés sur des sites, etc. Ceci est conforme à ce que nous trouvons dans notre corpus, car trois de nos quatre exemples proviennent de blogs.

Nature du habar kawn

Le second complément de kawn est majoritairement un syntagme nominal ou une phrase nominale. Il peut aussi consister en une phrase verbale et plus rarement en un syntagme prépositionnel.

- Le habar kawn est un nom, un syntagme nominal ou une phrase nominale :

Dans 53 \% des énoncés, le habar kawn est un syntagme nominal (un nom seul ou en annexion, un adjectif, un participe, etc.) ou une phrase nominale. En voici un exemple :

(24) Presse - Arabie Saoudite - Alwatan

fì kull șabāh 'inda hurūăğ-ì min al-manzil mutawağğihan 'ilāl-'amal 'udīru mawğat al-rādyū 'ilā mahațțat al-Kuwayt al-'id̄ā'iyya kawnu-hā l-'aqrab 'ilay-nā.

18. 'Ašadd min al-qatl est un emprunt coranique (Coran 2/191); la citation entre parenthèses est aussi coranique,

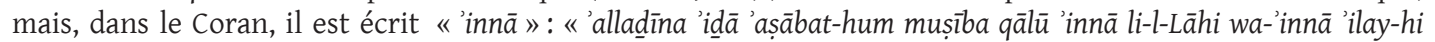
rāği üna » (Coran 2/156). Notez à la fin de l'extrait le mot muṣiba « coup du sort, calamité » faisant référence au même verset. 
Chaque matin, quand je sors de la maison pour aller au travail, je règle la fréquence de la radio sur la station des ondes koweitiennes parce qu'elles sont les plus proches de nous.

Le habar kawn est parfois introduit par un pronom sujet, comme ça peut être le cas dans une phrase nominale. Dans l'exemple suivant, on voit que l'effet produit est de mettre en relief le propos, mais aussi de permettre de bien interpréter le segment qui suit comme étant le habar kawn:

(25) Presse - Yémen - Almotamar

šaddada Yāsīn 'Abduh Sa'ìd 'udwu l-hay'a l-wațaniyya l-ulyā li-mukāfahat al-fasād 'alā 'ahammiyyat al-šarāka ma'al-muğtama' al-madan̄̄ wa-d̦aūrat al-'amal al-muštarak ma'al-munazzamāt ġayr al-hukūmiyya wa-l-šurakā’ al-duwaliyyīn fì mağāl mukāfahat al-fasād kawn hādihi l-qadiyya hiya qadiyyat muğtama:

De son côté, Yāsīn 'Abduh Saîd, membre de l'Autorité nationale suprême de lutte contre la corruption, a souligné l'importance du partenariat avec la société civile et la nécessité de travailler ensemble avec les ONG et les partenaires internationaux dans le domaine de la lutte contre la corruption, parce que cette affaire, c'est l'affaire d'une société.

(26) Blog - Egypte - Masryhorr

yaz̧har bi-wudūḥ 'anna l-mișriyyūn [sic] ğamîan ya lamūn kawn Isräài hiya 'adwu-hum al-'awwal.

Il apparaît clairement que tous les Égyptiens savent bien qu'Israël est leur principal ennemi, lit. « savent le fait que ».

En fait, ici, kawn est employé pour attester d'un fait: "les Égyptiens savent le fait que ». La même phrase avec 'an au lieu de kawn produit un effet plus neutre, alors que dans l'exemple (26) on instiste sur le fait qu'Israël est le principal ennemi des Égyptiens.

(27) Reformulation de (26)

Ya' lamūn 'anna Isrä'ìl hiya 'adwu-hum al-'awwal.

Ils savent qu'Israël est leur principal ennemi.

On constate bien avec la reformulation que kawn joue dans cet exemple le rôle d'introducteur de complétive, autrement dit celui d'un simple subordonnant.

- Le habar kawn est un syntagme prépositionnel :

Dans 4 \% des énoncés, le habarkawn est un syntagme prépositionnel.Il s'agit initialement d'une phrase nominale appelée «phrase nominale locative » ou "phrase nominale de localisation » dont le propos est un syntagme prépositionnel. Ceci dit, toutes les phrases que l'on nomme habituellement "phrases nominales de localisation » en référant à la structure inversée d'une phrase nominale (le propos, un syntagme prépositionnel, précède un thème indéterminé) n'indiquent pas une localisation spatiale, comme le montrent les exemples suivants :

(28) Presse - Syrie - Alwatan

... wa-hāsșsatan 'anna l-mufattiš huwa man yanqul al-șūra l-ḥaqiquiyya li-'amal al-mu'assasa li-kawni-hi 'alā-htikāk dā'im ma'a l-ummāl fì l-munšàāt. 
... surtout que l'inspecteur est celui qui véhicule la véritable image du travail de l'institution du fait qu'il est constamment en contact avec les travailleurs dans les établissements.

(29) Presse - Syrie - Tishreen

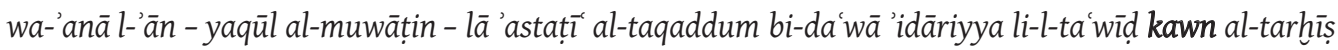
bi-sm al-muntafi.

Moi, maintenant- c'est le citoyen qui parle -je ne peux pas déposer une demande administrative d'indemnisation parce que le permis est au nom du bénéficiaire.

- Le habar kawn est une phrase verbale :

Dans $43 \%$ des énoncés, le second complément de kawn est une phrase verbale. Dans notre corpus, seul l'impératif ne figure pas; toutes les autres formes verbales ont été relevées. Toutes les formes verbales sont représentées à la forme positive ainsi qu'à la forme négative, de même qu'un inaccompli précédé de qad marquant le potentiel et de verbes au passif. Nous proposons des exemples pour chaque type. Après kawn, tous les temps peuvent figurer : on peut aussi bien trouver le passé (un verbe à l'accompli précédé ou non de qad), le présent (un verbe à l'inaccompli indicatif) ou le futur (un verbe à l'inaccompli indicatif précédé de sa-ou de sawfa) :

(30) Presse - Yémen - Almotamar

wa-bi-dāhilī nār mā yutffì-hā 'illā mufāraqat-ī li-l-hayāt kawn-ī faqadtu fildat kabid-ī wa-l-mutabaqqū lī min al-'usra kulli-hā.

À l'intérieur de moi [brûle] un feu que n'éteindra que mon abandon de la vie parce que j'ai perdu le fruit de mes entrailles et le restant de toute ma famille.

(31) Presse - Syrie - Tishreen

tamma saḥb al-intifā' 'an țarīq wizārat al-zirā'a kawn al-muntafi' hāalafa qānūn al-iṣlāh al-zirā'̄ wa-'ayḍan 'anna 'aqd al-bay' al-mawğūd bayna l-țarafayn huwa 'ayḍan muhālifli-l-qānūn.

La privation de l'usufruit a été opérée par le Ministère de l'Agriculture parce que le bénéficiaire avait contrevenu à la loi sur la réforme agraire, mais aussi parce que le contrat de vente entre les deux parties contrevenait lui aussi à la loi.

On remarque ici qu'un deuxième complément est coordonné. Il s'agit d'une proposition introduite par 'anna, ce qui nous renvoit aux exemples de kawn 'an/'anna vus ci-dessus (exemples 21 à 23). La présence du pronom de disjonction huwa au début du second habar kawn s'explique par la longueur de la phrase. Le fait que kawn soit coordonné à 'anna est un argument fort en faveur de notre hypothèse de grammaticalisation de kawn vers une particule servant à introduire une proposition avec une idée de lien de cause à effet.

(32) Blog - Syrie - Marcellita

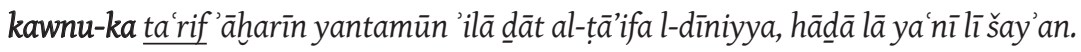

Le fait que tu connaisses d'autres personnes qui sont de cette confession, ceci ne veut rien dire pour moi.

(33) Blog - Yémen - Allielak

wa-l-ḍarūrì an tantaqid al-hayāt kawna-hā sa-tastamirr fì ğamî̀ al-ḥālāt.

Il est nécessaire que tu critiques la vie parce qu'elle se poursuivra dans tous les cas... 
Pour un exemple de futur introduit par sawfa, voir ci-dessus l'exemple (20). Les verbes peuvent être à la forme positive, comme dans les exemples que nous venons d'énumérer, ou à la forme négative :

(34) Blog - Arabie Saoudite - Mnsr

tu'îd 'asbāb al-hasāara 'ilā kawn al-lāibìn (li-l-'asaf) lam yatanāwalū mašrūb al-țāqa Rīd Būl qabl al-mubārāt.

Les causes de la défaite [du match] tiennent au fait que les joueurs (malheureusement) n'ont pas pris la boisson énergisante "Red Bull" avant le tournoi.

(35) Littérature - Liban - Najitahir

al-fān al-mutawaqqif' alā l-yasār, țalab min-nā sā'iqu-hu 'afrìtan yarfa'u-hu bi-hi, fa-tảassafnā li-kawn 'afrìti-nā lā yurakkab 'alā ġayr sayyārati-nā al-mahrūsa.

Le van arrêté sur la gauche, son conducteur nous a demandé un cric pour le lever, alors nous nous sommes excusés du fait que notre cric ne fonctionnait qu'avec notre voiture "protégée".

(36) Presse - Arabie Saoudite - Alwatan

'iktafā 'ahad al-mušārikīn bi-qawli-hi « hiya tawșiyyāt hağula, wa-sabab man' našri-hā li-kawni-hā lan tunaffad 'alā' ard al-wāqi' ka-gayri-hā min al-tawșiyyāt ».

L'un des participants s'est contenté de dire que « ce sont des recommandations timides, et la raison de l'interdiction de leur diffusion est due au fait qu'elles ne seront pas mises en œuvre sur le terrain comme toutes les autres recommandations ».

On peut aussi trouver un verbe au passif, comme dans l'exemple suivant ${ }^{19}$ :

(37) Presse - Yémen - Almotamar

wa-bi-'iğrā' mițl hādihi l-'amaliyyāt yuhaqqiq mustašfā l-țawra nuqla naw iyya mutaqaddima fì mağāal ğirāhat al-'izām wa-l-'amūd al-faqrī wa-llatī 'uğriyat bi-nağāh kawna-hā 'uğriyat li-'awwal marra fil-Yaman.

En ayant effectué de telles opérations, l'hôpital al-Tawra a réalisé une avancée qualitative dans le domaine de la chirurgie orthopédique et de la colonne vertébrale ; elles ont été couronnées de succès parce qu'elles ont été réalisées pour la première fois au Yémen.

Nous n'avons dans notre corpus qu'un seul habar kawn introduit par qad yakūn marquant une possibilité :

(38) Littérature - Liban - Najitahir

'aw rubbamā min 'išārati-hā ilā kawn al-'ahrāmāt qad takūn 'ibāra 'an nayāzik saqațat min al-samā' hunāk.

Ou peut-être par son allusion au fait que les pyramides seraient des sortes de météorites tombées du ciel à cet endroit.

On peut considérer comme un cas particulier de la phrase verbale les propositions, à l'origine nominales, qui sont modifiées par kāna ou l'une de ses « sœurs ». Nous avons dans notre corpus une phrase avec aṣbaḥa (devenir), trois phrases où il s'agit de mā zāla (ne pas

19. Voir aussi DiEM 1995, p. 52. 
cesser de) et huit autres où des propositions introduites par kāna constituent le habar kawn. Sept marquent le passé d'une phrase nominale, une le futur :

(39) Blog - Syrie - Wa2elblog

'atadakkar 'ihdāl-saharāt 'allatī ğama'at-nī bi-ba'd al-'așdiqā' mim-man yahlumūn bi-l-ḥuṣūl 'alā ğinsiyya 'ağnabiyya kawn ğinsiyyati-him al-sūriyya kānat 'ảiqan la-hum fìl-mațārāt wa-fì manāțiq wa-mawāțin 'uhrā.

Je me souviens d'une des soirées où j'étais réuni avec quelques amis qui rêvaient d'obtenir une nationalité étrangère parce que leur nationalité syrienne leur était un obstacle dans les aéroports et dans d'autres régions ou pays.

(40) Presse - Yémen - Almotamar

wa-tamannā fahāmat rảīs al-ğumhūriyya fì hitām muhāẹarati-hi li-muntasabī l-'akādīmiyya al-tawfīa

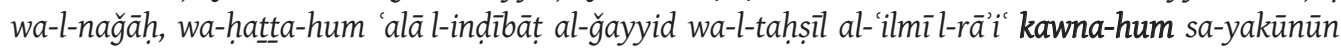
al-qudwa li-muntasabì l-quwwāt al-musallaḥa.

En clôture de sa conférence, le Président de la République a souhaité aux membres de l'Académie succès et réussite, et les a exhorté à une bonne discipline et à l'acquisition scientifique de haut niveau, parce qu'ils seront l'exemple pour les membres des forces armées.

L'ensemble de ces exemples montre que, si kawn garde la structure argumentale du verbe kāna bivalent, il ne conserve pas sa valeur temporelle, puisqu'il permet d'embrayer sur n'importe quel temps et sur tout type de forme verbale, active, passive, positive ou négative, ce qui est le propre d'un mașdar.

- Le habar kawn est une proposition introduite par 'an:

Nous avons relevé un exemple où le second complément de kawn est une proposition introduite par 'an:

(41) Presse - Syrie - Alwatan

kamā 'anna mu'zam ru'asā' l-wahadāt al-'idāriyya gayr mu'ahhalīn fanniyyan li-hādāa l-mansib li-kawn al-šarț al-'asāsī fì-stilāmi-him li-hādāa l-manșib 'an yakūnū muntahabìn.

Tout comme la plupart des présidents des unités administratives ne sont pas techniquement qualifiés pour cette fonction du fait que la condition essentielle dans leur prise de fonction est qu'ils soient élus.

L'explication pourrait être qu'il serait étrange, dans une phrase, que le mașdar kawn soit lui-même complément d'un autre maṣdar kawn. En effet, en second complément, on trouve souvent un maṣdar. On aurait donc théoriquement pu avoir « ... li-kawni l-šarți l-'asāsī fì-stilāmi-him li-hādā l-manșab kawna-hum muntahabina ». S'il s'agit là d'une possibilité pour le linguiste, le locuteur a préféré remplacer le complément par une proposition introduite par 'an al-mașdariyya, pour éviter une redondance malheureuse ou tout simplement parce qu'une proposition de ce type renforce la condition. 
Propriétés des compléments de kawn : mises en facteur commun

Tant le ism kawn (42) que le habar kawn (43) peuvent être mis en facteur commun, comme il est d'usage dans les annexions :

(42) Blog - Liban - Saudalmawla

'inna l-mubādara tanțaliqu min kawn al-țawā'if wa-l-ğamāa àt al-mukawwina li-l-muğtama' al-lubnānī, nawāfid haḍāriyya lā kuhūfan yahtabis fi-hā' atbā'u-hā.

L'initiative part du fait que les confessions et les groupes qui composent la société libanaise sont des fenêtres de civilisation, non des cavernes où s'enferment leurs adeptes.

(43) Blog - Yémen - Hoddar

wa-lā yahtimm 'abadan bi-madā kawni-hi... zawğ ğayyid [sic]... wa-'ab șālih [sic]... wa-fard [sic] dāa-ltizām muğtama:

Il ne s'intéresse jamais à l'importance qu'il soit... un bon mari... et un bon père... et un individu qui s'engage pour la communauté.

Insertion du syntagme dans la phrase

Les fonctions du syntagme

Les syntagmes de ce type sont susceptibles de remplir toutes les fonctions qu'un nom peut remplir en arabe. Parmi les exemples ci-dessus présentés, les syntagmes comprenant kawn jouent principalement le rôle de complément : ils peuvent entrer dans le champ d'une préposition pour former un complément de préposition $(18,22,28,34)$, avoir fonction de complément du nom (43), de complément du verbe (direct : 26 ; indirect : 21 , $34,35,38,42)$. Ils peuvent aussi être thème $(23,32)$ ou encore propos comme dans l'exemple suivant ${ }^{20}$ :

(44) Blog - Arabie Saoudite - Abuanas

wa-hādihi nuqța tuhsab li-l-wizāra wa-hiya rag̉batu-hum fì tahdị̂t al-bayānāt wa-kawnu-hum mā zālū yaḥtafizūn bi-'unwānī, wa-lākin al-mușība kānat fi tariqat taḥdịt al-bayānāt.

C'est un point à mettre à l'actif du ministère, à savoir leur volonté de mettre à jour les données, et le fait qu'ils conservaient encore mon adresse, mais la catastrophe c'était dans la manière de renouveler les informations!

Dans d'autres cas, kawn semble fonctionner comme une particule servant à coordonner deux propositions dont la seconde exprime la cause de la première (19, 24, 25, etc.). Certains verront dans la proposition introduite par kawna au cas direct un complément causatif.

20. Nous utilisons les termes «thème » et «propos » pour traduire les deux constituants de la phrase nominale chez les grammairiens arabes, respectivement le mubtadả et le habar. 


\section{Sémantique}

- Classification des emplois par valeur

Il convient maintenant d'opérer une classification des valeurs portées par kawn et des types d'emplois associés à ces valeurs. Des emplois de kawn, on peut dégager deux valeurs principales : le simple fait d'attribuer une qualité à une chose ou à une personne, et le fait que cette qualité soit la cause, la raison, l'explication d'un phénomène. Nous allons tenter de mettre à jour le lien entre ces deux valeurs, la seconde nous semblant découler de la première utilisée en composition avec les particules li- et bi- principalement.

1. Dans certains cas, nous avons constaté que kawn a uniquement le sens « lexical »du mașdar du verbe kāna bivalent (le fait d'être) ${ }^{21}$, comme dans l'exemple suivant:

(45) Blog - Syrie - Marcellita

kawn-ì'untāa 'awhama-ka 'anna-nì uhibb sațataka.

Le fait que je suis une femme t'a fait imaginer que j'aime ton impétuosité, lit. « mon fait d'être ».

Cet énoncé est la nominalisation de la tournure verbale :

(46) Reformulation de (45)

'an 'akūn 'unțā 'awhamaka 'anna-ni 'uhibb sațwataka.

Que je sois une femme t'a fait imaginer que j'aime ton impétuosité.

Du fait de la structure de la phrase où le syntagme kawn-ī untāa se trouve être le thème sujet du verbe awhamaka, on ne peut l'interpréter littéralement comme l'expression d'une cause, même si sémantiquement c'est bien « le fait d'être une femme » qui a «fait imaginer » à l'interlocuteur :

(47) Reformulation de (45)

* li-anna-nī' untā a awhamaka 'anna-nī uhibb sațataka.

* Parce que je suis une femme t'a fait imaginer que j'aime ton impétuosité.

C'est donc ce sens (le fait de/que) qu'il faut attribuer à kawn lorsqu'il se trouve après certaines particules dont le sens est incompatible avec l'idée de cause à effet. Dans notre corpus, nous avons huit occurrences de l'expression «bi-raǵmi kawni-hi » (malgré le fait que), deux autres occurrences après 'adā et après badala, comme dans la phrase suivante:

21. Pour nous, le fait que kawn puisse signifier à la fois « univers » et « le fait de » est à rattacher aux deux structures argumentales que le verbe kāna peut revêtir. Ainsi, pour le verbe bivalent, le mașdar signifie « le fait d'être », ce qui correspond lexicalement au verbe, mais son emploi est très souvent grammaticalisé. C'est pourquoi nous parlons d'emplois grammaticaux du mașdar dans ce cas, par opposition au mașdar lexical " univers ", même si « le fait d'être » est aussi la lexicalisation du verbe kāna, alors d'emploi bivalent. 
(48) Littérature - Maroc - Saidalkhiz

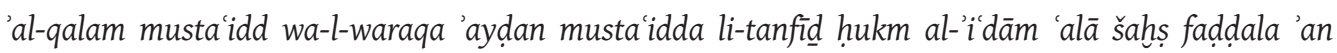
ya'ūd 'ilā l-hayāt li-yakūn ka-ğamí al-ašyā' 'aw li-yakūn 'ayy šay' badala kawni-hi 'insānan, himāran, hunfusā', ba'ū

Le crayon est prêt et la feuille aussi est prête pour exécuter la sentance de mort d'une personne qui a préféré retourner à la vie pour être comme toute chose ou pour être chaque chose au lieu d'être un homme, un âne, un cafard, un moustique, un mille-pattes...

On voit bien ici encore que kawn est paraphrasable par " 'an yakūn ». C'est aussi le cas dans les expressions "fadlan 'an kawn », " bi-șarf an-nazar 'an kawn » ou "bi-ġadd an-nazar 'an kawn» ("sans parler du fait que ») qui se répètent dans notre corpus cinq fois pour la première et trois fois pour chacune des deux autres.

2. Dans d'autres cas, au sens originel de kawn « le fait d'être » se voit ajoutée une valeur marquant la relation de cause à effet. Cette valeur est déduite de la syntaxe de la phrase ou induite par les particules bi- ou li-. Elle ne provient pas de kawn, mais du fait que celui-ci est employé pour introduire une cause :

(49) Blog - Syrie - Marcellita

kawn-ī' unțā ta' taqid 'anna-nī bi-l-fițra nāqișa wa-'anna-nī gabiyya wa-kull infíālāt-ī tudār bi-d̦̣irābāt hurmūniyya.

$\mathrm{Du}$ fait que/parce que je suis une femme, tu crois que je suis faible par nature, que je suis stupide et que tous mes actes sont régis par des perturbations hormonales.

Cet énoncé peut être comparé au (45), mais il fonctionne comme une sorte de « phrase double » : on déduit la relation de cause à effet de la première à la seconde. En inversant l'ordre des phrases, on pourrait parfaitement remplacer kawn par li-'anna («parce que ») ou par li-kawn:

(50) Reformulation de (49)

ta taqid 'anna-nī bi-l-fițra nāqișa (li-)kawn-ī'untāa.

Tu crois que je suis faible par nature du fait que/parce que je suis une femme.

(51) Reformulation de (49)

ta 'taqid 'anna-nī bi-l-fițra nāqișa li-'anna-ñ̄'untāa.

Tu crois que je suis faible par nature parce que je suis une femme.

On voit bien, en reformulant cette phrase, que l'ordre des propositions a son importance car elle implique clairement une interprétation de cause à effet. L'emploi de kawn seul pour marquer directement une relation de cause à effet est possible, mais cette relation est plus souvent marquée par les prépositions bi- et li-comme nous avons déjà pu le voir :

(52) Presse - Syrie - Alwatan

wa-ḩ̄șșatan 'anna 'ahadan min-hum lā yaltazim bi-ḍarūrāt al-wiqāya wa-l-amān mā ya'riḍu-hum

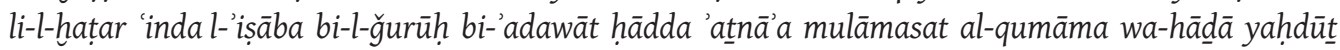
katîiran li-kawni-him yağhalūn muhtawā l-'akyās 'indamā yanbašūn bi-dāhili-hā bi-'aydī-him. 
Surtout qu'aucun d'entre eux ne se conforme aux nécessités de prévention et de sécurité qui les expose au risque d'infection des plaies en contact avec des objets pointus au cours du ramassage des ordures, ce qui se produit souvent du fait qu'ils/parce qu'ils ignorent le contenu des sacs dans lesquels ils plongent leurs mains.

Il apparait donc que li-kawn et bi-kawn fonctionnent comme des locutions subordinatrices marquant la cause. Ce sont syntaxiquement des syntagmes prépositionnels qui ont une fonction de compléments marquant la cause. Ces locutions peuvent être remplacées par li-anna, comme dans l'exemple suivant oùli-kawni-hā se trouve en coordination avec li-'anna-hā :

(53) Blog - Égypte - Window

tastahiqq'a àlā l-'awsima, laysa li-kawni-hā hașalat qabla šuhūr qalīla 'alāšahādat al-țānawiyya l-'āmma wa-hiya 'alà 'abwāb al-sittīn, wa-laysa li-'anna-hā -ltahaqat mu'ahharan bi-kulliyyat al-tarbiya

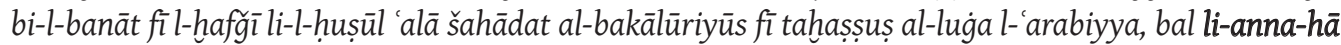
'umm 'ażìma anğabat 'ịhdā 'ašar ibnan wa-bna wa-ahsanat tarbiyata-hum fì zurūf șa'ba...

Elle mérite les plus hautes distinctions, non pas parce qu'elle a obtenu il y a quelques mois le baccalauréat avec près de $60 \%$, ni parce qu'elle a récemment rejoint la faculté d'éducation pour les filles à al-Hुafğì dans le but d'obtenir le baccalauréat spécialisé en langue arabe, mais parce que c'est une mère extraordinaire, qui a eu onze enfants et a su les bien élever dans des circonstances difficiles...

Dans notre corpus figurent de nombreuses occurrences de bi-kawn/li-kawn dans ce sens-là.

Jusque là, on peut imaginer que ces deux valeurs coexistent depuis toujours. D'ailleurs, on peut bien souvent paraphraser par li-'anna (" parce que ») et traduire par " parce que » et «du fait que ». Les deux emplois se confondent presque, la relation sémantique de cause à effet présente dans la phrase « colorant » l'emploi de kawn avec cette valeur causative. Mais là où le processus de grammaticalisation semble à l'œuvre, c'est lorsque la valeur de cause à effet est portée par kawn seul ou que ce dernier passe de substantif à simple subordonnant.

3. Ainsi, dans un second temps, il semble que l'on puisse réanalyser kawn survenant sans préposition comme conjonction de subordination marquant la cause. En effet, les kawn des phrases suivantes sont paraphrasables par li-anna :

(54) Presse - Liban - Assafir

wa-'aḍtarr yawmiyyan 'ilā sulūk al-țarīq, li-l-wușūl ilā Ṭarābulus, kawna-hā tahtașir al-waqt wa-l-masāfa, wa-lākin yawm ba'd yawm tatadāàaf al-mašākil bi-l-nisba 'ilay-nā.

Je suis contraint quotidiennement de suivre la route, pour arriver à Tripoli, parce qu'elle restreint le temps et la distance, mais jour après jour les problèmes nous concernant se multiplient...

(55) Littérature - Yémen - Bassamchamseddin

wa-habba li-munāșarati-hi l-șagìir wa-l-kabìr wa-dālika kawn 'abïhi 'akbar šayh fîl-madina.

Petits et grands volèrent à son secours, ceci parce que son père est le plus grand cheikh de la circonscription. 
Dans les exemples précédents, kawn apparaît comme complètement grammaticalisé car il est le lien formel entre la proposition principale et la subordonnée de cause. Il est paraphrasable par li-anna.

Dans d'autres cas, kawn devient un subordonnant neutre, sans valeur de cause. Certains emplois dans notre corpus préfigurent déjà cela et l'on peut imaginer que les emplois de ce type vont se multiplier. Observons la phrase suivante :

(56) Littérature - Arabie Saoudite - Jumanalahim

sanatān madatā... lam yakun yubālì kawn maktabi-hi' amām 'imārati-hi llatī'askana-hā'iḥdā šuqaqi-hā.

Deux ans passèrent... peu lui importait que son bureau soit en face de l'immeuble dont il était propriétaire et où il l'avait installée dans un appartement.

Il est intéressant de noter quel'auteur n'a pas employéla particule $b i$-après le verbe $b \bar{a} l \bar{a}$, probablement parce que bi-kawn est trop directement analysé comme introduisant une cause, alors qu'il s'agit dans cette phrase d'une simple complétive comme le montre le fait que l'on puisse remplacer kawn par 'anna:

(57) Reformulation de (56)

? lam yakun yubālī bi-kawn maktabi-hi 'amām 'imārati-hi...

Peu lui importait le fait que son bureau soit en face de son immeuble...

(58) Reformulation de (56)

? lam yakun yubālī bi-'anna maktaba-hu 'amām 'imārati-hi...

Peu lui importait que son bureau soit en face de son immeuble...

On peut placer dans cette catégorie "émergente » les occurrences de fikrat kawn "l'idée que ", récurrentes dans notre corpus, où la proposition introduite par kawn est complément du nom fikra et où kawn peut aussi être remplacé par 'anna:

(59) Blog - Liban - Trella

'alay-nā 'an nabdả fì l-tașāluh ma'a fikrat kawn hādihi taẓāhurāt ğāmi'a.

Nous devons commencer par nous concilier avec l'idée que ces démonstrations sont collectives...

- Étapes de la grammaticalisation du mașdar kawn

Avant d'avancer des hypothèses concernant les étapes de la grammaticalisation du mașdar kawn, il faut rappeler que trois emplois sont présents dans notre corpus : un emploi grammatical de la valeur lexicale du mașdar ( « le fait de »), un emploi en composition avec d'autres particules ou sans particule pour marquer la cause (« du fait que »; « parce que »), un emploi comme simple marqueur de subordination («que »). Certes, il ne s'agit pas ici d'une étude en diachronie, mais les occurrences présentes dans notre corpus permettent d'imaginer l'évolution de kawn. Il reste bien évidemment qu'une étude de linguistique historique serait nécessaire pour étayer ces hypothèses. Quoi qu'il en soit, la proportion assez réduite du troisième type d'emploi suggère qu'il est assez récent, alors que les deux 
premiers semblent coexister tous deux dans la langue de manière assez équilibrée et naturelle.

À la lumière de notre corpus, on peut imaginer l'évolution décrite dans le tableau ci-dessous, où figurent les différents emplois attestés du mașdar kawn ainsi qu'une proposition de paraphrase pour chacun d'eux ${ }^{22}$ :

\begin{tabular}{|c|c|c|}
\hline Valeurs de kawn & Emplois de kawn & Paraphrase \\
\hline $\begin{array}{l}\text { a. Kawn lexical « le fait d'être » conserve la structure argumentale } \\
\text { du verbe bivalent. Kawn en lui-même ne marque pas la cause. }\end{array}$ & $\begin{array}{l}\text { 1. kawn } \\
=\text { «le fait d'être }\end{array}$ & 'an yakūna \\
\hline $\begin{array}{l}\text { a'. (découlant directement de a.) Kawn conserve sa valeur lexicale } \\
\text { (le fait d'être) mais entre dans le champ d'une préposition : li-kawn/ } \\
\text { bi-kawn (par le fait que, du fait que), l'ensemble exprimant la cause. }\end{array}$ & $\begin{array}{l}\text { 1'. li-kawn/bi-kawn } \\
=\ll \text { du fait que » }\end{array}$ & li-'anna \\
\hline 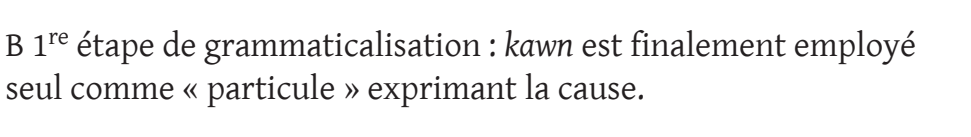 & $\begin{array}{l}\text { 2. kawn } \\
\text { «du fait que » } \\
=\text { «parce que » }\end{array}$ & li-'anna \\
\hline $\begin{array}{l}\text { c. } 2^{\mathrm{e}} \text { étape de grammaticalisation : kawn devient un subordonnant } \\
\text { neutre, introduisant une phrase nominale sans marquer la cause. } \\
\text { On peut imaginer que c'est vers cette valeur que kawn va évoluer et } \\
\text { que les emplois de ce type vont se multiplier. }\end{array}$ & $\begin{array}{l}\text { 3. kawn } \\
\text { = « que » } \\
\text { subordonnant }\end{array}$ & 'anna \\
\hline
\end{tabular}

Tableau 3 : Hypothèse de grammaticalisation du mașdar kawn à partir des exemples attestés.

À ce sujet, il est intéressant de noter que W. Diem (1995, p. 52) mentionnait bi-sabab parmi les différentes particules dans le champ desquelles kawn entre ${ }^{23}$. Il semble que l'on ait progressé dans la grammaticalisation de kawn, car les emplois combinés (particule - kawn) sont de moins en moins nombreux (hormis avec bi- ou li-) et il semble que l'idée de relation de cause à conséquence ait investi kawn, qui est de plus en plus utilisé seul dans ce sens. D’ailleurs, la plus grande liberté syntaxique générée par la grammaticalisation de kawn en conjonction de subordination de cause explique que l'on puisse retrouver à sa suite des propositions en anna.

Dans l'ouvrage qu'il consacre aux phénomènes de grammaticalisation des prépositions et subordonnants en arabe, M. Esseesy (2010) ne mentionne pas l'usage de kawn, ni en composition avec d'autres particules, ni comme subordonnant.

22. Les paraphrases sont toutes proposées à partir d'exemples où elles sont utilisées en coordination avec l'emploi de kawn.

23. Si nous ne retrouvons pas bi-sabab kawn (à cause du fait que) dans notre corpus, nous l'avons relevé dans la copie d'un étudiant arabophone lors d'un exercice de traduction du français vers l'arabe. 


\section{Forme et répartition des occurrences}

Sans tenir compte des valeurs portées par kawn, on peut observer la répartition entre les emplois de kawn seul ou en composition avec les particules li- et bi-.

\begin{tabular}{|c|c|c|}
\hline kawn & li-kawn & bi-kawn \\
\hline 201 & 53 & 14 \\
\hline
\end{tabular}

Tableau 4: Occurrences des emplois de kawn, li-kawn et bi-kawn.

La répartition générique des occurrences est très intéressante, car elle montre une très nette analogie entre les blogs et la presse, tous deux très éloignés de la littérature où les emplois grammaticaux de kawn sont environ trois fois moins nombreux.

\begin{tabular}{|c|c|c|}
\hline Blogs & Presse & Littérature \\
\hline 115 & 112 & 41 \\
\hline
\end{tabular}

Tableau 5 : Occurrences des emplois de kawn grammatical par genre.

La couverture diatopique est plus difficilement interprétable. Les exemples provenant de Syrie sont beaucoup plus nombreux du fait qu'un billet de blog répète beaucoup « kawn-ī». L'emploi moyen pour le Maroc, le Yémen, le Liban et l'Arabie Saoudite se situe autour de 44 occurrences. L'Égypte et plus encore la Tunisie sont nettement au-dessous de cette moyenne.

\begin{tabular}{|l|c|}
\hline \multicolumn{1}{|c|}{ Pays } & Occurrences \\
\hline Syrie & 60 \\
\hline Maroc & 53 \\
\hline Yémen & 47 \\
\hline Liban & 39 \\
\hline Arabie Saoudite & 37 \\
\hline Egypte & 24 \\
\hline Tunisie & 8 \\
\hline
\end{tabular}

Tableau 6: Occurrences des emplois de kawn grammatical par pays.

\section{Conclusion}

Cette photographie des emplois de kawn en arabe contemporain montre que trois valeurs différentes coexistent, par un phénomène de grammaticalisation que nous pourrions qualifier de lente et de non-exclusive. En effet, kawn dans le sens de "parce 
que » ne peut évincer l'emploi originel de kawn «le fait que » dont il découle. Il laisse cependant place à un degré de grammaticalisation supplémentaire où kawn est employé comme simple subordonnant.

Le rapport qu'il conviendrait d'interroger maintenant n'est pas celui des deux facettes de kawn (le fait de/parce que) mais bien plutôt celui du rapport du subordonnant kawn aux autres subordonnants et particules marquant la cause (li-'anna " parce que ", bi-mā 'anna «du fait que », bi-sabab «à cause de », etc.) ou introduisant une subordonnée ('an/'anna «que »). Il serait aussi intéressant d'observer quel usage il est fait de kawn dans différents dialectes. Une étude de linguistique historique permettrait quant à elle de quantifier les emplois grammaticaux de kawn, afin de pouvoir vérifier si ceux-ci sont plus nombreux en arabe contemporain qu'à l'époque classique.

Pour ce qui est de déterminer les éléments qu'il faudrait retenir de cette description et qui pourraient intégrer une grammaire d'arabe moderne, il nous semble que li-kawn, bi-kawn mais aussi kawn seul pourraient figurer dans la liste des particules marquant la cause aux côtés de li-'anna. En revanche, l'emploi de kawn comme subordonnant n'est à notre avis pas assez attesté pour pouvoir figurer comme emploi courant dans une grammaire. 


\section{Bibliographie}

\section{Sources}

Ibn al-Ḥāğib, Ğamāl al-Dīn, Al-Imlā' 'alāl-Kāfiya fĩl-naḥw, éd. Manuel SARTORI, in Le Šarh al-Kāfiya de Ibn al-Hāğib: édition critique d'un manuscrit grammatical arabe $d u \mathrm{VII}^{e} / \mathrm{XIII}^{e}$ siècle, thèse de doctorat inédite, Université Aix-Marseille, 2012.

Les autres sources classiques mentionnées, dans les notes 3 et 4 , sont accessibles en ligne sur le site d'al-Maktaba al-šāmila : http://shamela.ws.

\section{Études}

BADAWI El said, CARTER Michael et GULLY Adrian 2004, Modern Written Arabic. A comprehensive Grammar, London/New York, Routledge (collection Comprehensive Grammars).

BUCKWALTER Timothy et PARKINSON Dilworth 2011, A Frequency Dictionary of Arabic. Core Vocabulary for learners, Londres et New-York, Routledge.

DiEM Werner 1995, «Kawnuhu rasūlan "weil er Bote ist" und Verwandtes. Ein Beitrag zur Syntax des nachklassischen Arabisch », Zeitschrift der Deutschen Morgenländischen Gesellschaft 145/1, p. 49-105.

ESSEESY Mohssen 2010, Grammaticalization of Arabic Prepositions and Subordinators. A corpus-based study, Leiden, Brill (Studies in Semitic Languages and Linguistics, vol. 59).

FISCHER Wolfdietrich 2002 [1971], Grammatik des klassichen Arabisch, Wiesbaden, Harrasowitz Verlag (Porta Linguarum Orientalium), $3^{\mathrm{e}}$ édition revue et corrigée.

ĠALĀYĪNĪ (al-), Muștafā 1973 (12 édition), Ǧāmi‘ al-durūs al-'arabiyya, Beyrouth/Saida, al-Maktaba al-'așriyya.

PINON Catherine 2011, « La grammaire arabe : entre théories linguistiques et applications didactiques », Synergie-Monde arabe 7, p. 75-86.

[En ligne] http://ressources-cla.univ-fcomte.fr/gerflint/Mondearabe7/mondearabe7.html.

PINON Catherine 2012 a, « Les enjeux épistémologiques et didactiques d'une grammaire arabe fondée sur corpus », in Teddy ARNAVIELLE (éd.), Voyages grammairiens, Paris, L'Harmattan, p. 83-101.

PINON Catherine 2012 b, «La nébuleuse de kān: classification des différents emplois de kāna : yakūnu à partir d'un corpus d'arabe contemporain », thèse de doctorat inédite, Université Aix-Marseille.

PINON Catherine 2013 a, «Quel corpus peut aider à fonder la grammaire d'une langue pluriglossique? Exemple de l'arabe contemporain », Cahiers de Praxématique 54-55 (Corpus, données, modèles), p. 39-58.

PINON Catherine 2013 b, «Les valeurs de kâna en arabe contemporain », Romano-Arabica XIII (Arab Linguistic, Literary and Cultural Studies), p. 305-322.

SILVESTRE DE SACY Antoine-Isaac 1810, Grammaire arabe à l'usage des élèves de l'école spéciale des langues orientales vivantes, Paris, Imprimerie impériale.

VAN Mol Mark 2003, Variation in Modern Standard Arabic in Radio News Broadcasts. A Synchronic Descriptive Investigation in the Use of Complementary Particles, Louvain, Peeters (Orientalia Lovaniensia Analecta 117).

\section{Dictionnaires}

Munğid fíl-lugia l-mu'āṣira (al-), 2006 (2 éd.), Beyrouth, Dār al-Mašriq. 